\title{
STOCHASTIC RESONANCE IN A BROWNIAN RATCHET
}

\author{
ANDREW ALLISON and DEREK ABBOTT \\ Centre for Biomedical Engineering (CBME) and EEE Dept., \\ Adelaide University, SA 5005, Australia \\ Email: aallison@eleceng.adelaide.edu.au,dabbott@eleceng.adelaide.edu.au
}

Received 31 October 2001

Revised 17 January 2002

Accepted 22 January 2002

\begin{abstract}
We present evidence to support the idea that Stochastic Resonance (SR) and Brownian Ratchets (BR) share underlying physical principles. We examine the special case of a discrete-time ratchet, called Parrondo's games, and show that the addition of noise increases the rate of flow in the ratchet up to a certain point, after which the addition of further noise causes the rate of flow to decrease. We argue that the rate of flow of particles in a $\mathrm{BR}$ is analogous to the rate of flow of information in the case of SR.
\end{abstract}

Keywords: Stochastic resonance; Brownian ratchet; Parrondo's games.

\section{Introduction}

The etymological root of the word "noise" is mooted to be the Latin word noxia, to hurt, or possibly: nausea, meaning disgust or sickness. The very concept of noise involves the idea of something harmful or unpleasant. This is certainly the traditional attitude to noise in electrical circuits. The Shannon-Hartley-Tuller law [1] states that

$$
C=B \log _{2}\left(1+\frac{S}{N}\right)
$$

where $C$ is the maximum mean information rate of the channel in bits per second, $B$, is the bandwidth in hertz, $S$ is the average signal power in watts and $N$ is the random noise power in watts. The channel capacity of a linear system is clearly limited by noise. The flow of information through the channel decreases uniformly as the noise power is increased. However, when the system is nonlinear, noise can play a constructive role. This is clear in the cases of Brownian Ratchets (BR) and Stochastic Resonance (SR). It is possible that these are really two completely unrelated phenomena that only appear to be related because of the use of the word of "constructive" to describe both systems. Alternatively, there may be common 
underlying physical principles in both phenomena. The distinction between these two very different possibilities is an important open question.

Some authors Bier [2,3], Berdichevsky \& Gitterman [4] and Astumian \& Moss [5] have all alluded to a connection between SR and BR. Abbott [6] has noted that asymmetry is present in both situations and has postulated that any common mechanism must depend on asymmetry. Gammaitoni et al. [7] have pointed out that stochastic resonance can be described in terms of the solution to the Fokker-Planck equation:

$$
\frac{\partial}{\partial t} p(x, v, t ; \phi)=\left\{-\frac{\partial}{\partial x} v+\frac{\partial}{\partial v}\left[\gamma v+f(x)-A_{0} \cos (\Omega t+\phi)\right]+\gamma D \frac{\partial^{2}}{\partial v^{2}}\right\} p(x, v, t ; \phi)
$$

where the variables are as defined in [7]. The Fokker-Planck equation is also used by many authors, including Doering [8], to analyze Brownian ratchets. It is quite possible that a general connection between SR and BR may be established through a careful analysis of the Fokker-Planck equation. More information about the methods of solution and the applications of the Fokker-Planck equation can be found in Risken [9].

At the present time, the authors cannot find any reference in the literature which directly connects SR and BR through a mathematical formalism. In this paper, we demonstrate stochastic resonance in the special case of a discrete-time Brownian ratchet, called Parrondo's games.

\section{Brownian Ratchets and Parrondo's Games}

A Brownian ratchet is a particular type of heat engine, in which the Brownian motion of small particles is controlled and directed using a modulated field. Real Brownian ratchets have been constructed and have been found to work [10-14]. Parrondo's games $[15,16]$ are a pair of games that were devised by Parrondo to provide a very simple illustration of the principles of the flashing Brownian ratchet [17]. These games were originally derived from a flashing Brownian ratchet $[16,18,19]$.

The games are labelled as Game "A" and Game "B". They have the curious property that an indefinite sequence of Game A is losing; an indefinite sequence of Game B is losing but randomized sequences of Games A and B, such as $\{A B B A B \cdots\}$, are winning. It is possible to non-deterministically combine two "losing" games together to produce a "winning" result! This counter-intuitive behavior is possible because the games have internal states.

In the original formulation, the conditional probabilities of winning or losing depend on the state, $k$, of capital but not on any other information about the past history of the games:

- Game A is a toss of a biased coin:

$$
p_{\text {win }}=\frac{1}{2}-\epsilon
$$

where $\epsilon$ is an adverse external bias that the game has to "overcome". This bias, $\epsilon$, is typically a small number such as $\epsilon=1 / 200$, for example $[18,19]$. 
- Game B depends on the capital, $k$ :

If $(k \bmod 3)=0$, then the odds are unfavorable.

$$
p_{\text {win }}=\frac{1}{10}-\epsilon
$$

If $(k \bmod 3) \neq 0$, then the odds are favorable.

$$
p_{w i n}=\frac{3}{4}-\epsilon
$$

It is straightforward to simulate a randomized sequence of these games on a computer using a very simple algorithm [16].

There are a number of ways to explain the behavior of Parrondo's games. The "Boston Interpretation" views it as a noise-induced phenomenon as follows: Game $\mathrm{B}$ loses when played alone, because the $k \bmod 3$ internal state biases the game towards the unfavorable branch in (4). The random mixture of Game A in with Game B acts as noise that breaks up the internal state and tilts the bias towards the favorable branch in (5). This has been mathematically formalized by Harmer et al. in [16] and it is the noisy behavior of Game A that we shall exploit in our demonstration of stochastic resonance.

\section{Stochastic Resonance}

Stochastic Resonance (SR), [7,20,21] is a phenomenon where a signal can be enhanced by noise. In these systems, the addition of some noise to the input actually increases the available information at the output. Generally, the signal is considered to be auto-correlated and has a non-zero auto-correlation function for at least some finite time delay. The system is generally considered to have stable modes or at least thresholds. Recent work by Bezrukov and Vodyanoy [22] suggests that thresholds or barriers may not actually be necessary. The outer limits of what constitutes SR may be quite wide and are not yet completely determined [6].

To show that Parrondo's games exhibit SR, we need to show that the relationship between added noise, due to Game $\mathrm{A}$ and the time-rate of change in capital, $k$, has the appropriate form. This was achieved using a simulation in Matlab. We created a parametric graph of the return from the randomized Parrondo process as a function of increased noise power due to the presence of Game A. This is shown in Fig. 1 and clearly has the form of a stochastic resonance curve.

The solid line represents the theoretical prediction for the time-averaged process.

The games were formulated as discrete-time finite Markov chains. This naturally gives rise to matrix formulation of the games:

$$
\mathbf{v}_{\mathbf{t}+\mathbf{1}}=\mathbf{v}_{\mathbf{t}} \cdot\left[c_{i, j}\right]
$$

where $\mathbf{v}_{\mathbf{t}}$ represents the time varying probability vector, at time $t$, and $C=\left[c_{i, j}\right]$ represents the time-averaged game, $C=\gamma A+(1-\gamma) B$, and $\gamma$ is the probability of selecting Game A. The individual coefficient $c_{i, j}$ is the conditional probability that the player will be in state $k=j$ after the next round of the games given an 


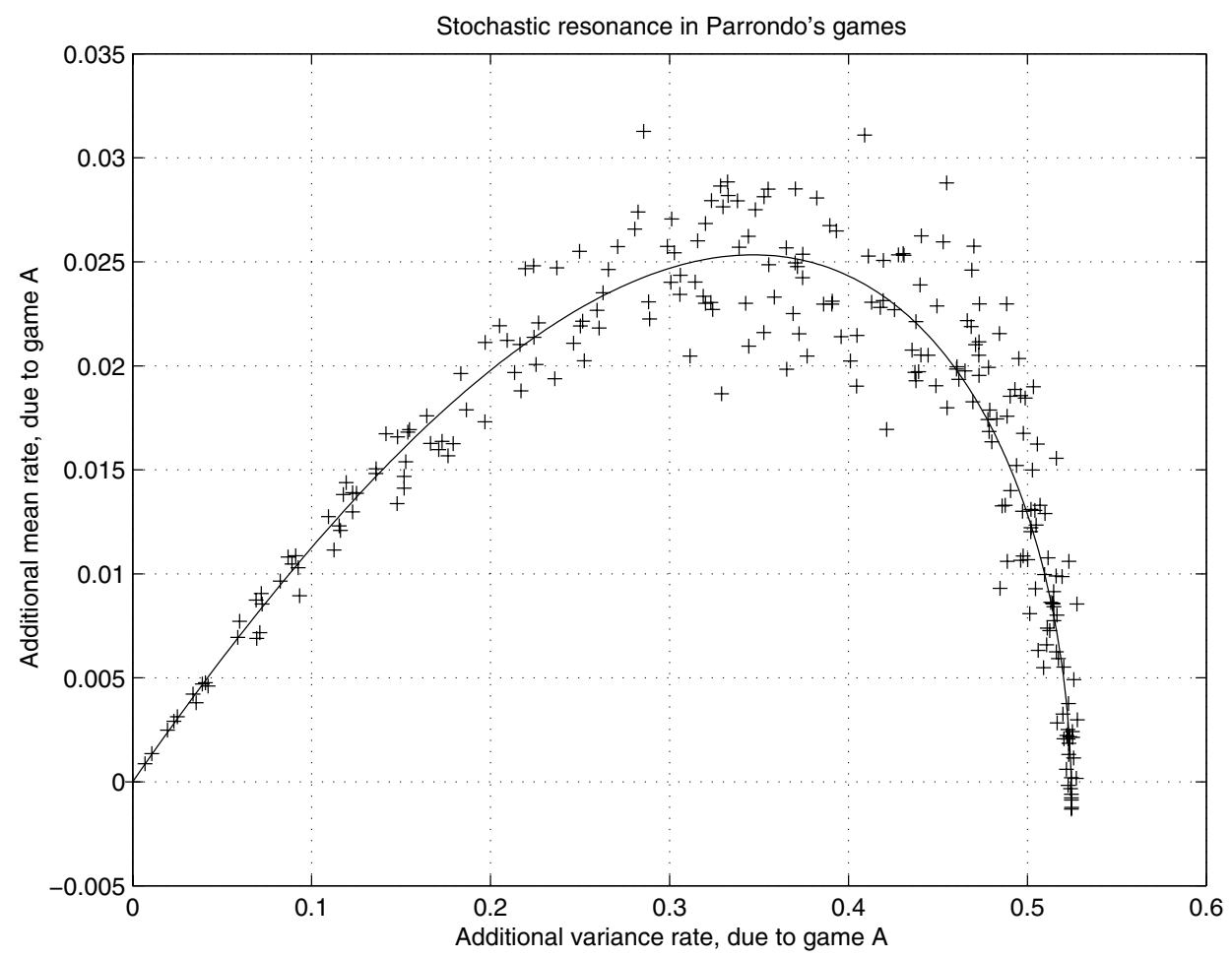

Fig. 1. The parametric relationship between variance and return. As more of Game A is added to Game B there is a dual effect. Game A adds more noise to the process and increases the time-rate of increase of the variance, or noise power. The addition of more Game A also reduces the dependency of the whole process on the mod3 states of Game B and allows the barriers in Game B to be penetrated. Too much Game A will eventually degrade performance. This leads to the characteristic SR curve, shown above. The Y coordinate were numerically calculated using Eq. (9). The X coordinate was defined in terms of Eq. (10). It is not the mixing parameter $\gamma$. It is the difference in the rate of increase in variance due to the presence of game $\mathrm{A}$. This is equivalent to a diffusivity or, alternatively, to the noise power contributed by the presence of game A.

initial condition of state $k=i$. The simple matrix Eq. (6) follows from the laws of conditional probability. The matrices $A$ and $B$ are defined in the same way.

The moments required for Fig. 1 were evaluated numerically by calculating the time-varying probability vector, $\mathbf{v}_{\mathbf{t}}$, and calculating the relevant weighted sums. More explicitly, the first moment is defined as:

$$
\mu=\sum_{k=-\infty}^{+\infty} k \cdot v_{k}
$$

and the central second moment, or variance, is defined as

$$
\sigma^{2}=\sum_{k=-\infty}^{+\infty}(k-\mu)^{2} \cdot v_{k}
$$


The rates of change of these moments were estimated in terms of finite differences:

$$
\dot{\mu} \approx \frac{\mu(t+\Delta t)-\mu(t)}{\Delta t}
$$

and

$$
\dot{\sigma^{2}} \approx \frac{\sigma^{2}(t+\Delta t)-\sigma^{2}(t)}{\Delta t}
$$

The rates of change in the moments were used to draw the parametric curve for the solid line in Fig. 1. The scattered data points in Fig. 1 were the results of direct simulations of Eq. (3), Eq. (4) and Eq. (5). The sampled points are the results from finite simulations.

We place the following interpretation on the results:

- The signal in this case is the potential rate of return from the "good" part of Game B.

- The barrier in this case is the "bad" part of Game B which leads to a certain distribution of states which prevents a winning result.

- The noise in this case is the "randomness" added by Game A.

- Noise allows the penetration of the barriers.

- Barrier penetration allows an increased rate of return.

- One can visualize Game B as a 3-state Markov chain, with detailed balance at $\epsilon=0$. By introducing the random behavior of Game $\mathrm{A}$, the resulting drift in capital is clearly a case of noise-induced breaking of detailed balance.

- The asymmetry of the system is embodied in the structure of Game B.

\section{Conclusions}

Bier [2,3], Berdichevsky [4] and Astumian \& Moss [5] have previously suggested that there is an connection between Brownian ratchets and stochastic resonance. We demonstrate an actual quantitative stochastic resonance curve in a particular Brownian ratchet example for the first time.

The most important open problem is to generalize this relationship to include all classes of Brownian ratchet. Given that Parrondo's games are really a particular way of sampling the Fokker-Planck equation and that stochastic resonance and Brownian ratchets can be represented in terms of solutions to the Fokker-Planck equation, then it is likely that the general solution to the open problem will come from a careful consideration of the properties of the Fokker-Planck equation.

\section{Acknowledgments}

The authors like to acknowledge funding from the Australian Research Council and the GTECH Corporation Australia. 


\section{References}

[1] L. Brillouin, Science and Information Theory, Academic Press Inc., New York (1956).

[2] M. Bier, Brownian ratchets in physics and biology, Contemp. Phys. 38 371-379 (1997).

[3] M. Bier, A motor protein model and how it relates to stochastic resonance, Feynman's ratchet, and Maxwell's demon, Stochastic Dynamics 386 81-87, Springer, Berlin (1997).

[4] V. Berdichevsky and M. Gitterman, Stochastic resonance and ratchets - New manifestations, Phys. A 249 (1998) 88-95.

[5] R. D. Astumian and F. Moss, Overview: The constructive role of noise in fluctuation driven transport and stochastic resonance, Chaos 8(3) (1998) 533-538.

[6] D. Abbott, Overview: Unsolved problems of noise and fluctuations, Chaos 11(3) (2001) $526-538$.

[7] L. Gammaitoni, P. Hanggi, P. Jung and F. Marchesoni, Stochastic resonance, Reviews of Modern Physics 70(1) (1998) 223-287.

[8] C. R. Doering, L. A. Dontcheva and M. M. Klosek, Constructive role of noise: Fast fluctuation asymptotics of transport in stochastic ratchets, Chaos 8(3) (1998) 643-649.

[9] H. Risken, The Fokker-Planck Equation, Springer, Berlin (1985).

[10] L. P. Faucheux, L. S. Bourdieu, P. D. Kaplan and A. J. Libchaber, Optical thermal ratchet, Phys. Rev. Lett. 74(9) (1995) 1504-1507.

[11] G. W. Slater, H. L. Guo and G. I. Nixon, Bidirectional transport of polyelectrolytes using self-modulating entropic ratchets, Phys. Rev. Lett. 78(6) (1997) 1170-1173.

[12] D. Ertas, Lateral separation of macromolecules and polyelectrolytes in microlithographic arrays, Phys. Rev. Lett. 80(7) (1998) 1548-1551.

[13] T. A. Duke and R. H. Austin, Microfabricated sieve for the continuous sorting of macromolecules, Phys. Rev. Lett. 80(7) (1998) 1552-1555.

[14] J. S. Bader, R. W. Hammond, S. A. Henk, M. W. Deem, G. A. McDermott, J. M. Bustillo, J. W. Simpson, G. T. Mulhern and J. M. Rothberg, DNA transport by a micromachined Brownian ratchet device, PNAS 96(23) (1999) 13165-13169.

[15] G. P. Harmer and D. Abbott, Parrondo's paradox: Losing strategies cooperate to win, Nature 402 (1999) 864.

[16] G. P. Harmer, D. Abbott, P. G. Taylor and J. M. R. Parrondo, Brownian ratchets and Parrondo's games, Chaos 11(3) (2001) 705-714.

[17] C. R. Doering, Randomly Rattled Ratchets, Il Nuovo Cimento 17D(7-8) (1995) 685697.

[18] G. P. Harmer and D. Abbott, Parrondo's paradox, Statistical Science 14(2) (1999) 206-213.

[19] G. P. Harmer, D. Abbott and P. G. Taylor, The paradox of Parrondo's games, Proc. Roy. Soc. Lond. A 456 (1994) (2000) 247-259.

[20] F. Moss and K. Wiesenfeld, The Benefits of Background Noise, Scientific American 273(2) (1995) 50-53.

[21] D. G. Luchinsky, R. Mannella, P. V. E. McClintock and N. G. Stocks, Stochastic resonance in electrical circuits: I Conventional stochastic resonance, IEEE Transactions on circuits and systems 46(9) (1999) 1205-1214.

[22] S. M. Bezrukov and I. Vodyanoy, In search of a possible statistical basis of stochastic resonance, Proc. 2nd Int. Conf. on Unsolved Problems of Noise and fluctuations (UPoN'99), eds. D. Abbott and L. B. Kiss, American Institute of Physics, Adelaide, Australia. Vol. 511 (2000) 169-178. 